Helical core tokamak MHD equilibrium states

This article has been downloaded from IOPscience. Please scroll down to see the full text article.

2011 Plasma Phys. Control. Fusion 53124005

(http://iopscience.iop.org/0741-3335/53/12/124005)

View the table of contents for this issue, or go to the journal homepage for more

Download details:

IP Address: 128.178.125.164

The article was downloaded on 15/11/2011 at 08:18

Please note that terms and conditions apply. 


\title{
Helical core tokamak MHD equilibrium states
}

\author{
W A Cooper, J P Graves, O Sauter, J Rossel, M Albergante, S Coda, \\ B P Duval, B Labit, A Pochelon, H Reimerdes and the TCV team
}

Ecole Polytechnique Fédérale de Lausanne (EPFL), Centre de Recherches en Physique des Plasmas, Association Euratom-Confédération Suisse, CH1015 Lausanne, Switzerland

E-mail: wilfred.cooper@epfl.ch

Received 24 June 2011, in final form 25 July 2011

Published 14 November 2011

Online at stacks.iop.org/PPCF/53/124005

\begin{abstract}
Bifurcated magnetohydrodynamic (MHD) tokamak equilibrium states with axisymmetric or helical core structure are computed. When a peaked pressure profile is chosen, the helical core structures appear like the snakes that are observed in the JET tokamak. They also have the allure of saturated ideal internal kinks. The existence of a magnetic island is not a requisite condition. Novel equilibrium states that can model the snake are obtained for a JET configuration when the $q$-profile has weak reversed magnetic shear with minimum $q$ values in the range 0.94 to 1.03 . At the lower end of this $q_{\min }$ range, the equilibrium snake structure lies radially well inside the domain for which $q_{\min } \leqslant 1$. Free boundary equilibria computed for the TCV tokamak develop helical cores when $\beta_{\mathrm{N}}$ exceeds 0.3 and have a significant axis excursion for $\beta_{\mathrm{N}} \geqslant 0.4$. At fixed $\langle\beta\rangle=1.6 \%$, the distortion of the magnetic axis is large in the range $0.95 \leqslant q_{\min } \leqslant 1.01$. The plasma-vacuum interface is not significantly altered by the internal helical deformations.
\end{abstract}

(Some figures in this article are in colour only in the electronic version)

\section{Introduction}

Internal three-dimensional (3D) structures have been extensively observed in tokamak devices and are generally attributed to magnetohydrodynamic (MHD) instability. Many of these can exist for very long times, even for almost a whole discharge. Among such examples are the snakes in JET [1,2], Tore Supra [3] and other machines, the continuous modes that persist in TCV well after the disappearance of sawteeth [4,5], the long-lived modes in MAST [6], the saturated ideal internal kinks in NSTX [7] and the change of sawteeth from kink-like to quasi-interchange-like in DIII-D [8]. Another important development is the single helical axis (SHAx) configurations obtained in the RFX-mode reversed field pinch [9]. It is our contention that these represent essentially the same physical phenomenon and in this work we explore 
the proposition that the 'instability' structures observed in the experiments we have alluded to constitute in reality novel equilibrium states with $3 \mathrm{D}$ character.

Investigations of nonlinearly saturated $m=1, n=1$ ideal internal kinks have been pursued both analytically [10-12] and numerically with the application of large scale, computationally intensive codes [13-15]. Bifurcated equilibrium states in tokamaks were first obtained by adding a forcing term to the second variation of an energy principle, then removing it to get a static solution with perturbations around rational surfaces that were interpreted as indications of the formation of magnetic islands [16]. More global structures like the SHAx equilibria were computed with the application of the VMEC code $[17,18]$ in the reversed field pinch RFX-mod with seven-fold toroidally periodic structures [19]. In tokamak systems with an imposed axisymmetric boundary, internal helical equilibrium states similar to saturated ideal $m=1, n=1$ kink modes have been calculated using the ANIMEC code [20], a modified version of the VMEC code [18], in model configurations of the TCV tokamak [21], ITER [22], MAST [23] and JET [24]. In this paper, we extend the work in [24] to yield more detailed information about the simulation of snake-like equilibria in JET and we present initial free boundary computations that model bifurcated equilibrium states in the TCV tokamak.

In section 2, we outline a brief review of 3D toroidal MHD equilibrium theory. In section 3, we investigate fixed boundary JET equilibria with peaked pressure profile to simulate snake conditions. In section 4 , we identify a coil configuration current set that is predicted to lead to free boundary bifurcated equilibria in the TCV tokamak. Finally, a conclusion and discussion section outlines the main results of this paper.

\section{3D MHD equilibrium theory}

The determination of the MHD equilibrium in 3D magnetic confinement systems is treated such that nested magnetic flux surfaces are imposed. Thus magnetic islands and stochastic regions are excluded from consideration. The energy $W$ of the system is defined as

$$
W=\iiint \mathrm{d}^{3} x\left(\frac{B^{2}}{2 \mu_{0}}+\frac{p_{\|}}{\Gamma-1}\right),
$$

and we solve the inverse equilibrium problem, namely we determine $R=R(s, u, v)$ and $Z=Z(s, u, v)$, where $0 \leqslant s \leqslant 1$ is the radial variable corresponding to the normalized enclosed toroidal magnetic flux, $0 \leqslant u \leqslant 2 \pi$ is the poloidal angle and $0 \leqslant v \leqslant 2 \pi / L$ is the toroidal angle in which $L$ is the number of field periods. We vary the energy with respect to an artificial time parameter $t$ conserving the plasma mass and the magnetic fluxes. This leads to an equation of the form

$$
\begin{aligned}
\frac{\mathrm{d} W}{\mathrm{~d} t}= & -\iiint \mathrm{d} s \mathrm{~d} u \mathrm{~d} v\left[F_{R} \frac{\partial R}{\partial t}+F_{Z} \frac{\partial Z}{\partial t}+F_{\lambda} \frac{\partial \lambda}{\partial t}\right] \\
& -\iint_{s=1} \mathrm{~d} u \mathrm{~d} v\left[R\left(p_{\perp}+\frac{B^{2}}{2 \mu_{0}}\right)\left(\frac{\partial R}{\partial u} \frac{\partial Z}{\partial t}-\frac{\partial Z}{\partial u} \frac{\partial R}{\partial t}\right)\right] .
\end{aligned}
$$

The last term corresponds to the forces that move the plasma-vacuum interface (by definition it vanishes in fixed boundary calculations). Note that by choosing $\partial R / \partial t=F_{R}, \partial Z / \partial t=F_{Z}$ and $\partial \lambda / \partial t=F_{\lambda}$, the equation acquires negative definite form and is thus amenable to solution by minimization techniques. The vanishing of $F_{\lambda}$ corresponds to the condition of current lines lying on flux surfaces. The components $F_{R}$ and $F_{Z}$ can be shown to correspond to the projections $\sqrt{g} \nabla v \times \nabla Z \cdot \boldsymbol{F}$ and $\sqrt{g} \nabla R \times \nabla v \cdot \boldsymbol{F}$ of the force $\boldsymbol{F}=-\boldsymbol{\nabla} \cdot \boldsymbol{P}+\boldsymbol{j} \times \boldsymbol{B}$. The pressure tensor $\boldsymbol{P}$ is chosen in the form [25] $\boldsymbol{P}=p_{\perp} \boldsymbol{I}+\left(p_{\|}-p_{\perp}\right) \boldsymbol{b} \boldsymbol{b}$ where $p_{\|}\left(p_{\perp}\right)$ is the pressure parallel (perpendicular) to the magnetic field lines, $\boldsymbol{b}=\boldsymbol{B} / \boldsymbol{B}$ is the unit vector 
along the magnetic field lines, $\boldsymbol{I}$ is the identity matrix and $\sqrt{g}$ is the Jacobian. The binormal force projection $\sqrt{g} \boldsymbol{B} \times \nabla s \cdot \boldsymbol{F} / B^{2}=-F_{\lambda}$, where $\lambda$ is the poloidal angle renormalization parameter [17]. Fourier decomposition in the periodic angular variables $u$ and $v$ together with a special finite difference scheme for the radial discretization is applied. An accelerated steepest descent energy minimization method is invoked with matrix preconditioning to iteratively obtain the equilibrium state $[17,18]$. The radial force balance equation is employed as a diagnostic for the quality of the equilibrium state that is attained. This is given by

$$
\left\langle\frac{F_{s}}{\Phi^{\prime}(s)}\right\rangle=-\left\langle\left.\frac{1}{\Phi^{\prime}(s)} \frac{\partial p_{\|}}{\partial s}\right|_{B}\right\rangle-\frac{\partial}{\partial s}\left\langle\frac{\sigma B_{v}}{\sqrt{g}}\right\rangle-\iota(s) \frac{\partial}{\partial s}\left\langle\frac{\sigma B_{u}}{\sqrt{g}}\right\rangle,
$$

where the toroidal magnetic flux function is $\Phi$, prime $\left(^{\prime}\right)$ indicates the derivative of a flux surface quantity with respect to $s,\langle\cdots\rangle$ denotes a flux surface average, $p_{\|}$simplifies to the thermal pressure $p(s)$ for the calculations performed in this work which leads to $\sigma=1 / \mu_{0}$. The rotational transform is $\iota(s)=1 / q, B_{u}$ and $B_{v}$ are the poloidal and toroidal magnetic fields in the covariant representation, respectively, and $F_{s}$ is the radial force (in covariant representation). This model is implemented in the ANIMEC code [20].

\section{Simulations of JET snake equilibria}

The standard description of the snake relies on pellet material ablated in the interior of a $q=1$ island that is partially maintained by an influx of impurities and improved local confinement that increases the density [26]. There are, however, some consistency problems with this model. In the experiments, snakes survive sawtooth events $[1,2]$ that would presumably remove the $q=1$ rational surface from the plasma. Furthermore, in Tore Supra, the snake radius is considerably smaller than that of the $q=1$ island [3]. These observations make it difficult to reconcile the existence of the snake within the confines of the $q=1$ magnetic island. We propose an alternative description associated with the development of a novel equilibrium state with the allure of a saturated ideal $m=1, n=1$ internal kink. These type of kink modes have been shown to be nonresonantly destabilized in a circular axisymmetric tokamak when the value of $q_{\min }$ is in the range of unity to 1.014 [27]. We shall discuss our perspective for snake formation later in this text.

The boundary of JET is described by $R_{\mathrm{b}}=R_{0}+a \cos (\theta+\delta \sin \theta)$ and $Z_{\mathrm{b}}=E a \sin \theta$ with $R_{0}=2.96 \mathrm{~m}, a=1.25 \mathrm{~m}, E=1.68$ and $\delta=0.3$. For the calculations we undertake, the boundary remains fixed. We choose as input the plasma mass and the toroidal current profile. The resulting pressure profile has an edge pedestal (typical of H-mode tokamak operation) and is peaked on axis. The toroidal current profile corresponds to piecewise continuously differentiable polynomials, namely a quadratic function with respect to $s$ in the centre of the plasma, a cubic function near mid-radius and a linear function towards the edge of the plasma [28]. The resulting $q$-profiles for the cases considered with different toroidal currents are shown in figure 1 . Here $2 \pi J$ denotes the total toroidal current.

In the range of toroidal currents $2 \pi J=3.77 \mathrm{MA}$ to $4.05 \mathrm{MA}$, the minimum value of $q$ $\left(q_{\text {min }}\right)$ drops from $\sim 1.025$ to $\sim 0.945$. In this domain, we obtain bifurcated equilibrium solutions with the ANIMEC code. The local minimum energy solution obtained depends on the initial guess provided for the position of the magnetic axis. A sufficiently helically deformed initial value evolves towards the helical core branch result. Otherwise, the axisymmetric branch ensues. Examining the pressure distribution at the midplane as a function of $R$ and $v$, we see that at high current a small snake structure distinctly emerges. It becomes significantly larger and more extended when $q_{\min }$ is in the neighbourhood of unity and decreases in size again when $q_{\text {min }}$ approaches 1.02 as demonstrated in figure 2 . The case with toroidal current 


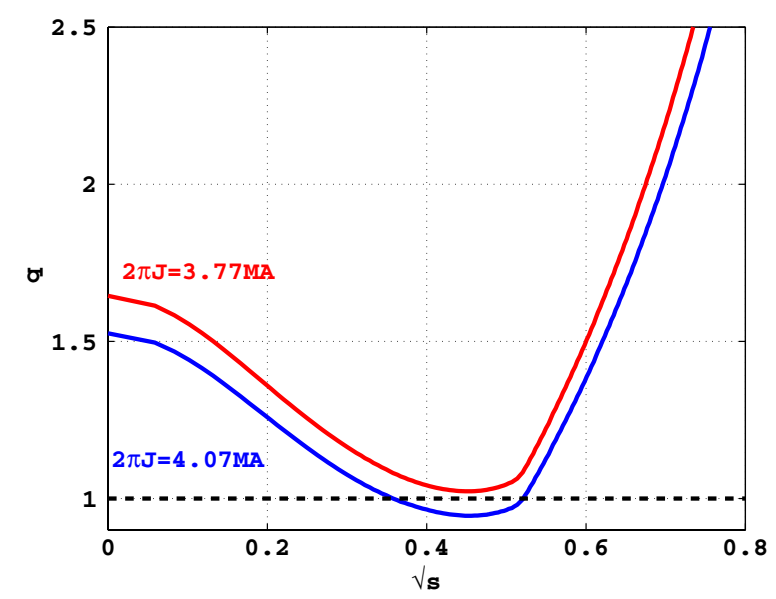

Figure 1. The inverse rotational transform $q$-profiles that encompass the domain for which bifurcated solutions of the MHD equilibrium state are realized as a function of $\sqrt{s}$ in JET.
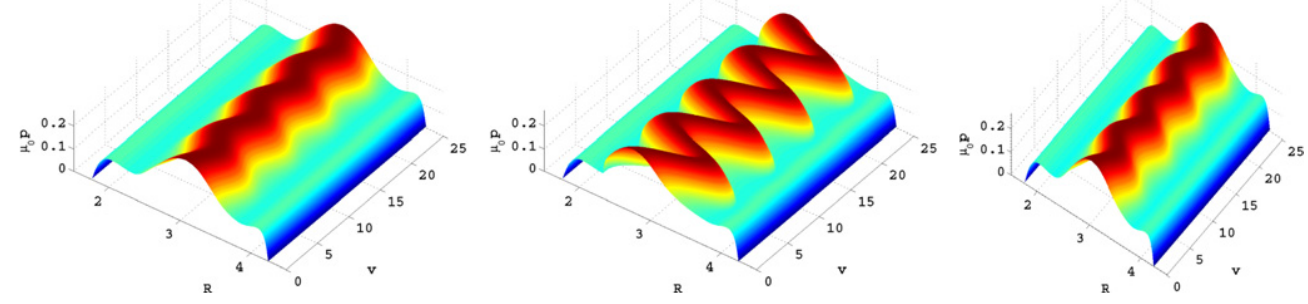

Figure 2. The pressure distribution (multiplied by $\mu_{0}$ ) in JET as a function of $R$ and $v$ at the midplane for $2 \pi J=3.77 \mathrm{MA}, q_{\min } \simeq 1.023$ (left), for $2 \pi J=3.95 \mathrm{MA}, q_{\min } \simeq 0.995$ (middle) and for $2 \pi J=4.07 \mathrm{MA}, q_{\mathrm{min}} \simeq 0.945$ (right). The pressure thus is given in units of pascals times $\mu_{0}=4 \pi \times 10^{-7} \mathrm{H} / \mathrm{m}$, the permeability of free space.

$2 \pi J=4.07$ MA which corresponds to $q_{\text {min }} \simeq 0.945$ merits particular interest. In figure 3 , we superimpose the rotational transform $1 / q$ and the pressure (multiplied by $5 \mu_{0}$ ) distributions at the plasma midplane as functions of $R$ and $v$. In addition, we plot a cut of the $q=1$ plane in this domain. This clearly shows that the pressure distortion (the darker red surface) lies radially inside the range for which $q \leqslant 1$ (the lighter orange surface). We assert that this is consistent with the observations of the snake location with respect to the $q=1$ surface position reported on Tore Supra [3].

The fractional energy difference between the helical branch solution and its axisymmetric counterpart is very small, only a fraction of a per cent, even smaller than $\langle\beta\rangle \simeq 2.3 \%$ in the case examined. At higher currents $(2 \pi J=3.93 \mathrm{MA})$ where $q_{\min }<1$, the helical branch has slightly lower energy. The converse holds when $q_{\min } \geqslant 1$. This is displayed in figure 4 . The helical excursion of the magnetic axis $\delta_{\mathrm{H}}$ is plotted as a function of $q_{\mathrm{min}}$ in figure 5 which shows bifurcated equilibrium solutions in the range $0.94<q_{\min }<1.03$ with a maximum distortion that occurs at $q_{\text {min }}$ slightly below unity. We have also performed a linear ideal MHD stability analysis of the axisymmetric branch solutions with the TERPSICHORE code [29]. The growth rates (multiplied by a factor of 10 for graphical convenience) as a function of $q_{\text {min }}$ are also shown in this figure and their magnitudes align very well with the axis deformation 


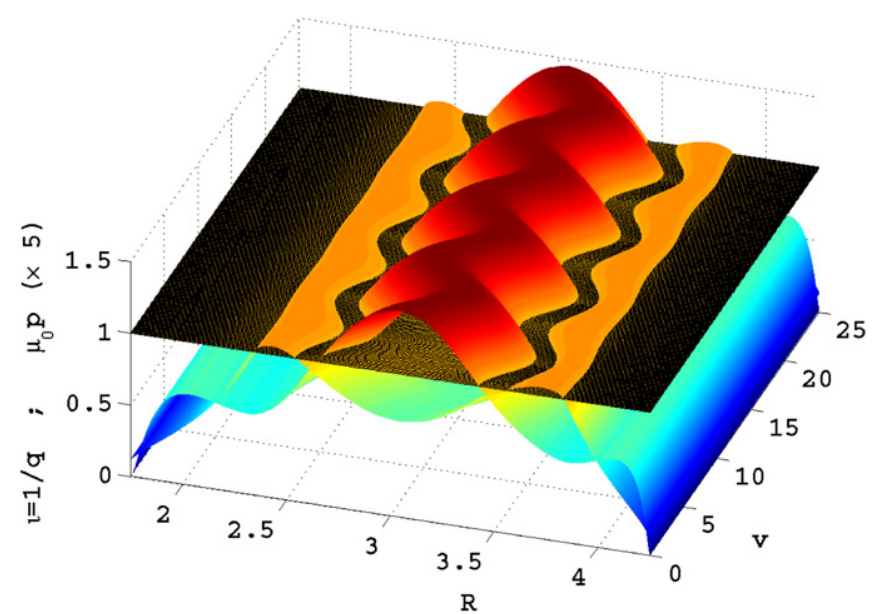

Figure 3. The pressure distribution (dark red in the core) and the $\iota=1 / q$ distribution in JET as functions of $R$ and $v$ at the midplane for the case with $2 \pi J=4.07 \mathrm{MA}$ and $q_{\min } \simeq 0.945$. The darker plane that cuts the pressure and $\iota$ surfaces corresponds to $q=1$. The pressure deformation (in dark red) is localized radially well inside the domain for which $q \leqslant 1$ (in orange). Note that we have multiplied the pressure by $5 \mu_{0}$.

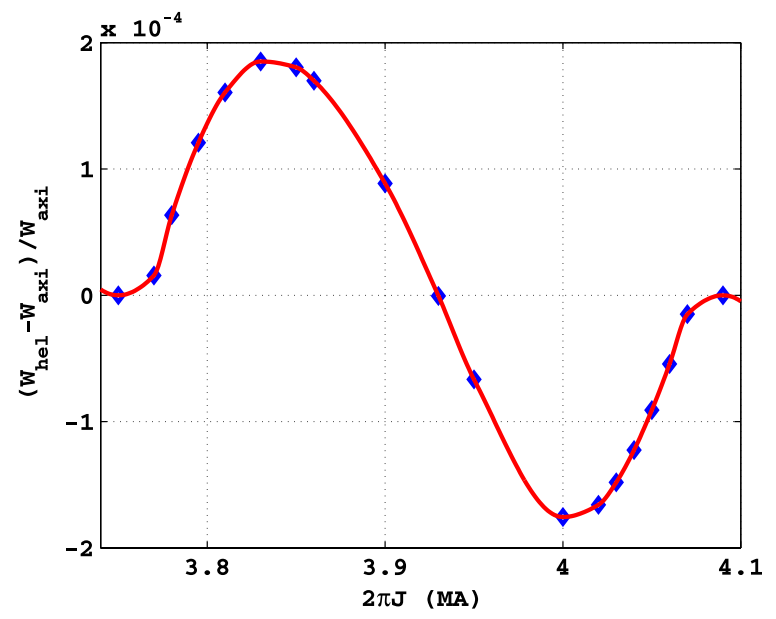

Figure 4. The fractional energy difference between the helical and axisymmetric bifurcated branch solutions in JET as a function of the toroidal plasma current.

$\delta_{\mathrm{H}}$ of the helical branch solutions, particularly when $q_{\min }>1$. The growth rates remain finite for $q_{\min }<0.94$ due to the persistence of the internal kink mode [30].

\section{Free boundary TCV equilibrium states}

Free boundary calculations of MHD equilibria for the TCV tokamak require the determination of the vacuum magnetic fields in the configuration. To achieve this, the current in the toroidal and poloidal field (PF) coils must be specified. We model each of the 16 toroidal coils with a single filament carrying a current of $358 \mathrm{kA}$. The $16 \mathrm{PF}$ coils, which are closer to the plasma, 


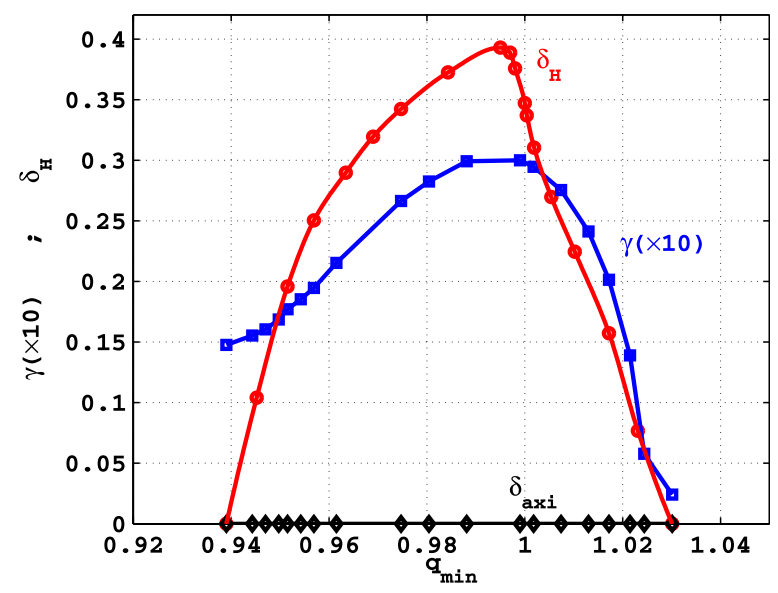

Figure 5. The helical axis excursion $\delta_{\mathrm{H}}$ for the helical branch bifurcated equilibrium solution, the excursion of the axis for the axisymmetric branch $\delta_{\text {axi }}$ (by definition vanishing) and the linear ideal growth rate of the internal kink mode in the axisymmetric branch $(\times 10)$ as functions of $q_{\min }$ in JET. $\left.\delta_{\mathrm{H}} \equiv \sqrt{(} R_{01}^{2}+Z_{01}^{2}\right) / a$, where $R_{01}\left(Z_{01}\right)$ is the $m=0, n=1$ Fourier component of $R(Z)$ and $a$ is the minor radius.

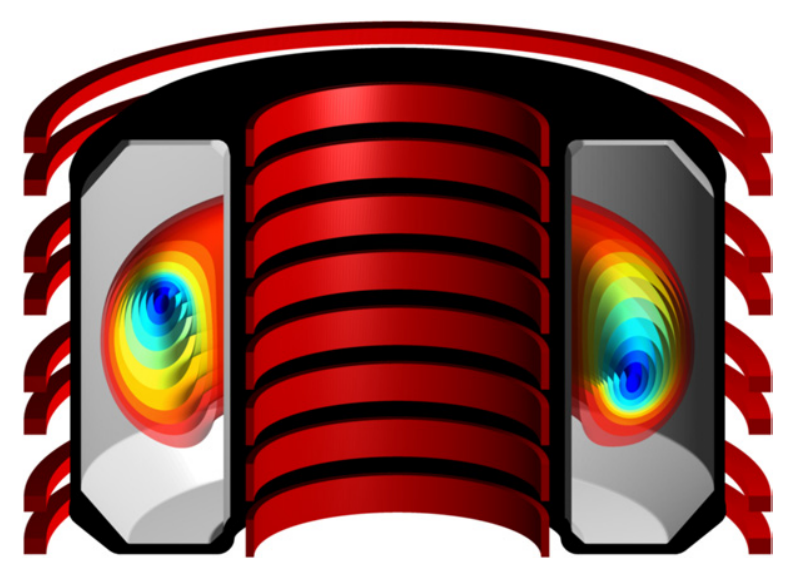

Figure 6. The TCV PF coils, vacuum vessel and pressure contours of the helical solution of a bifurcated equilibrium state that encompasses half of a toroidal transit.

are modelled with 4 filaments each. The inner PF coils above the midplane have currents of $-60 \mathrm{kA},-40 \mathrm{kA},-8 \mathrm{kA}$ and $167 \mathrm{kA}$ from top to bottom, respectively. The corresponding outer PF coil currents are $40 \mathrm{kA}, 48 \mathrm{kA}, 52 \mathrm{kA}$ and $64 \mathrm{kA}$ from top to bottom. We consider up-down symmetric plasma configurations, thus the PF coil currents specified are reflected about the midplane for the set in the lower half of the torus. The Biot-Savart law is applied to obtain the vacuum magnetic fields from this ensemble of current filaments. Figure 6 shows the PF coils, the vacuum vessel and the pressure surfaces of a bifurcated free boundary TCV equilibrium solution covering half the torus.

We have prescribed the pressure with the nearly parabolic profile $p(s)=p(0)(1-s)(1-$ $\left.s^{4}\right)$. The toroidal current profile is prescribed as

$$
\begin{aligned}
2 \pi J^{\prime}(s)=0.98 & +5.168 s-67.464 s^{2}+440.42 s^{3}-1391.7 s^{4}+2146.5 s^{5}-1582.1 s^{6} \\
& +448.27 s^{7},
\end{aligned}
$$




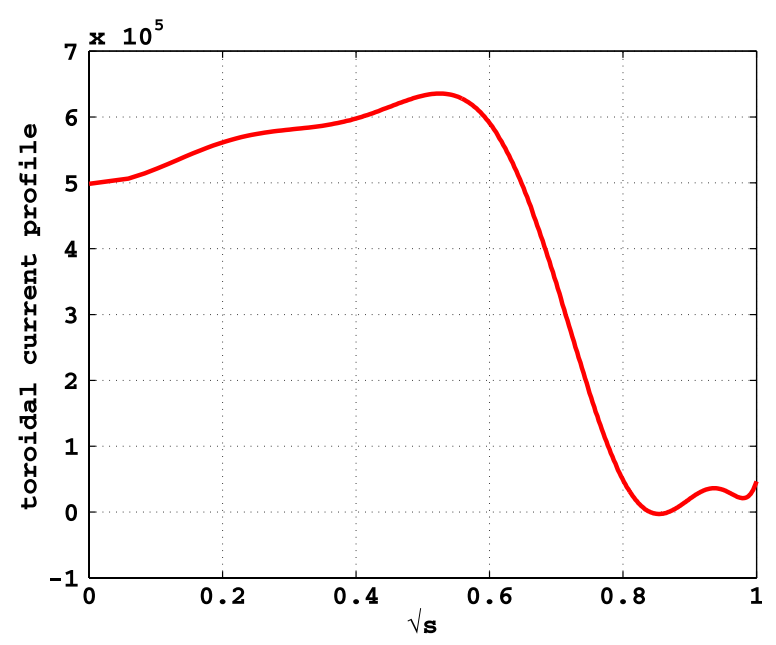

Figure 7. The toroidal current profile that is prescribed for TCV equilibrium computations as a function of $\sqrt{s}$.

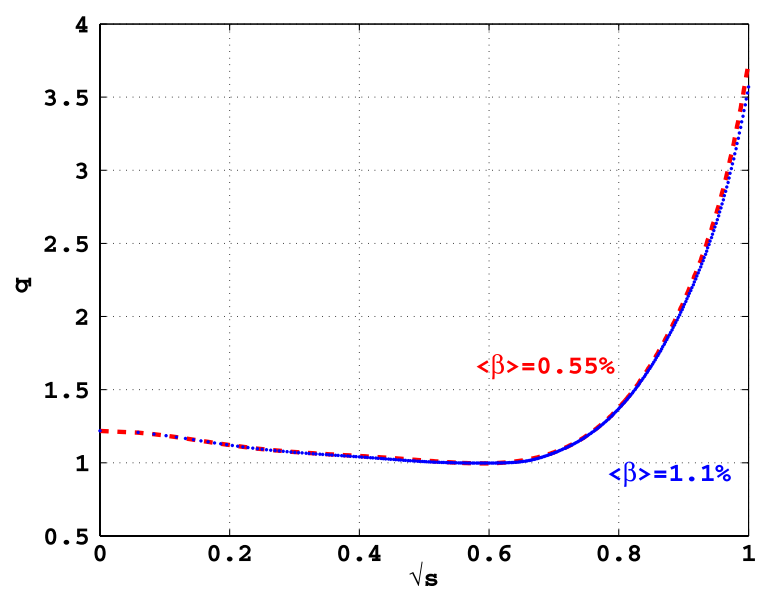

Figure 8. The inverse rotational transform $q$-profiles at $\langle\beta\rangle=0.55 \%$ and $\langle\beta\rangle=1.1 \%$ as a function of $\sqrt{s}$ in the TCV bifurcated equilibrium calculations.

and is displayed in figure 7. It is preferable to use the toroidal current rather than the $q$-profile to avoid unphysical reversed edge currents. The resulting $q$-profiles at $\langle\beta\rangle=0.55 \%$ and at $\langle\beta\rangle=1.1 \%$ are plotted in figure 8 . Only small differences are apparent mainly near the edge of the plasma.

At $\langle\beta\rangle=0.55 \%$, the bifurcated solution branches merge and the plasma is basically axisymmetric. However, at $\langle\beta\rangle=1.1 \%$, two distinct solutions emerge. The pressure distribution for the virtually axisymmetric solution at $\langle\beta\rangle=0.55 \%$ and that of the helical branch at $\langle\beta\rangle=1.1 \%$ are displayed in figure 9 at 4 cross sections covering half of a toroidal transit with toroidal angles $v=0, \pi / 3,2 \pi / 3$ and $\pi$, respectively.

The pressure distribution $\left(\mu_{0} p\right)$ as a function of $R$ and $v$ at the midplane for $\langle\beta\rangle=0.55 \%$, $\langle\beta\rangle=0.9 \%$ and $\langle\beta\rangle=1.6 \%$ is presented in figure 10 .

The corresponding values of $\beta_{\mathrm{N}}=\langle\beta\rangle a B_{\mathrm{t}} / I_{\mathrm{t}}$ (where $B_{\mathrm{t}}$ is the toroidal magnetic field, $I_{\mathrm{t}}=2 \pi J(s=1)$ is the toroidal plasma current) are $\beta_{\mathrm{N}}=0.33,0.54$ and 0.98 , respectively. 

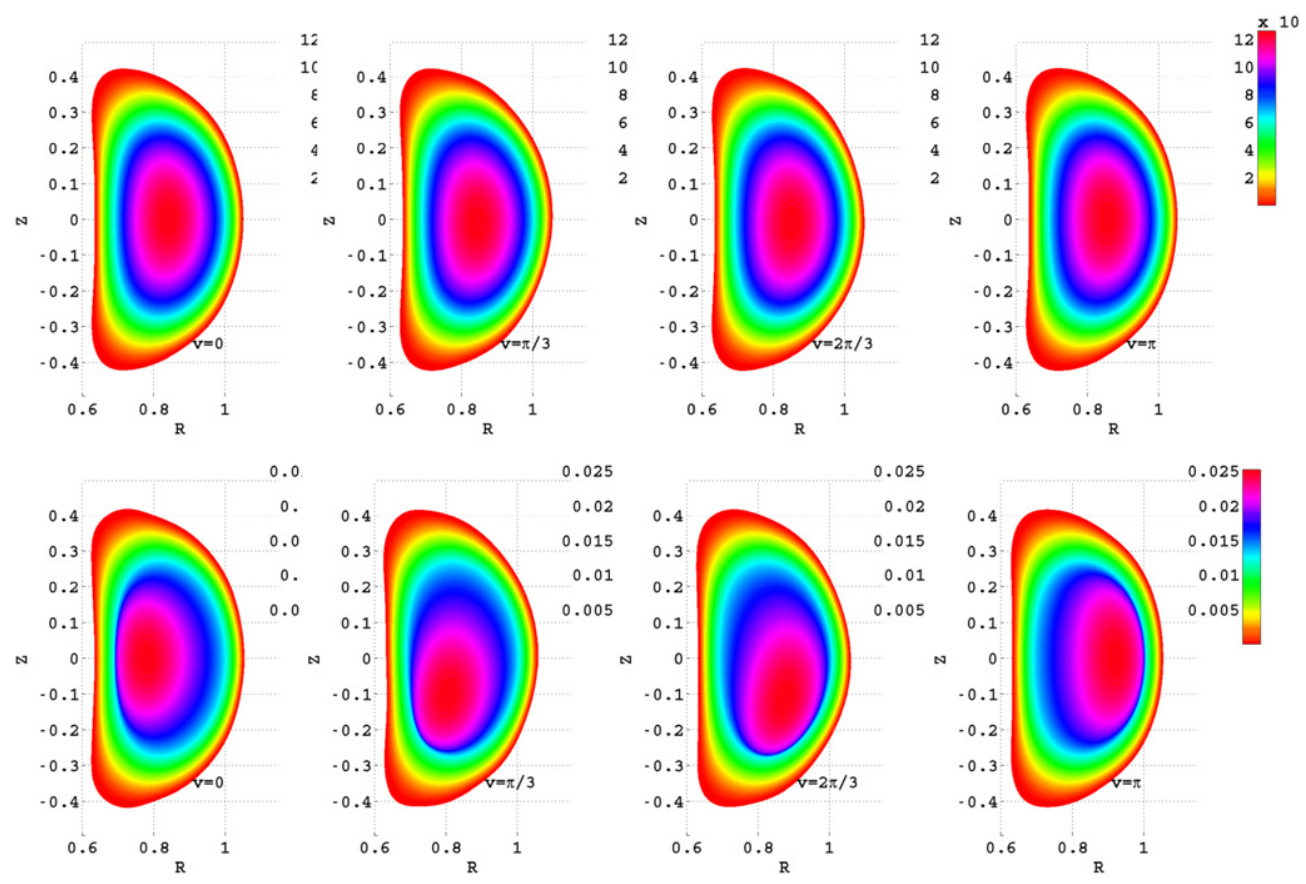

Figure 9. Contours of constant pressure at the cross sections with toroidal angle $v=0, v=\pi / 3$, $v=2 \pi / 3$ and $v=\pi$ (columns from left to right) for the axisymmetric branch solution at $\langle\beta\rangle=0.55 \%$ (top row) and for the helical branch equilibrium solution at $\langle\beta\rangle=1.1 \%$ (bottom row), where $R$ and $Z$ are expressed in metres.

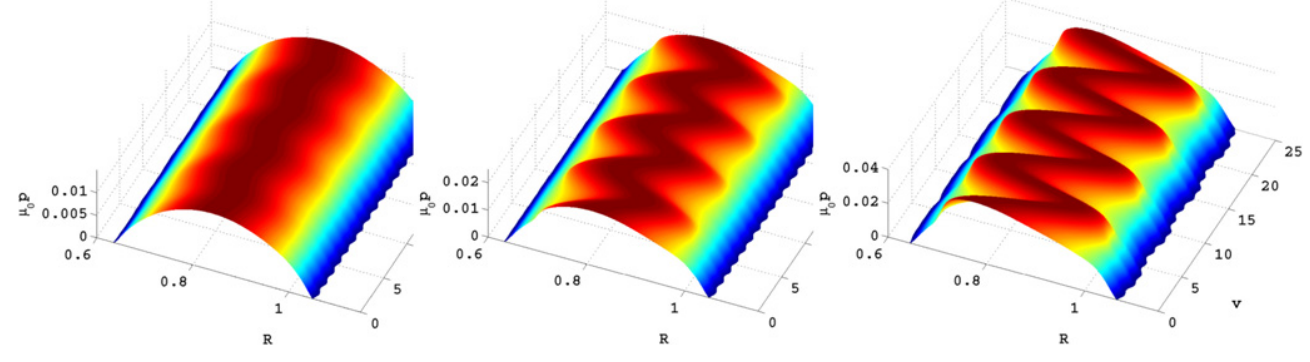

Figure 10. The pressure distribution $\mu_{0} p$ at the midplane as a function of $R$ and $v$ at $\langle\beta\rangle=0.55 \%$ (left), at $\langle\beta\rangle=0.9 \%$ (middle) and at $\langle\beta\rangle=1.6 \%$ (right).

This figure shows an incipient deformation of the plasma core at $\beta_{\mathrm{N}}=0.33$, which becomes much more significant at $\beta_{\mathrm{N}}=0.54$ and resembles a strong snake structure at $\beta_{\mathrm{N}}=0.98$. One important point to note here is that the helical distortions remain concentrated in the internal part of the plasma. The wiggles near the plasma-vacuum interface can be wholly attributed to the ripple induced by the discreteness of the toroidal field coils.

In figure 11, we quantify the helical excursion of the magnetic axis of the TCV helical branch solutions we have computed in free boundary with the variation of $\langle\beta\rangle$ by plotting $\delta_{\mathrm{H}}$ as a function of $\beta_{\mathrm{N}}$. It shows that the helical branch develops at $\beta_{\mathrm{N}} \sim 0.3$, that the axis distortion grows to about $60 \%$ of the minor radius at $\beta_{\mathrm{N}} \sim 0.8$ and saturates for higher $\beta_{\mathrm{N}}$. At nearly constant $\langle\beta\rangle=1.6 \%$, we show the variation of $\delta_{\mathrm{H}}$ with respect to $q_{\mathrm{min}}$ in figure 12 . 


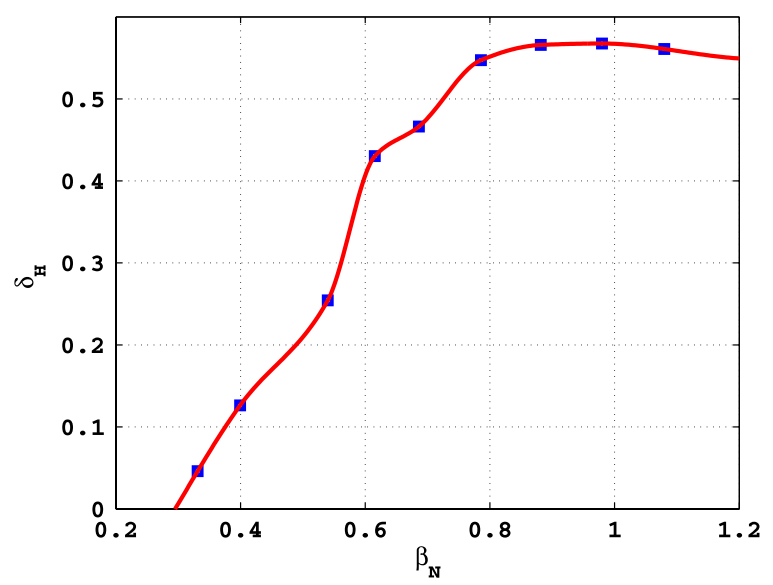

Figure 11. The magnetic axis helical distortion parameter $\delta_{\mathrm{H}}$ as a function of $\beta_{\mathrm{N}}$ with toroidal current $2 \pi J=488 \mathrm{kA}$.

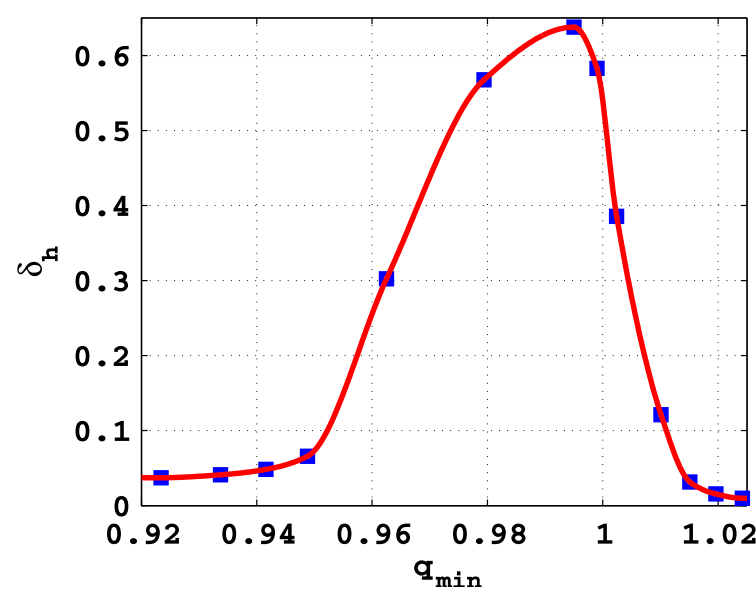

Figure 12. The magnetic axis helical distortion parameter $\delta_{\mathrm{H}}$ as a function of $q_{\min }$ at $\beta_{\mathrm{N}}=0.98$. The toroidal current varies from 475 to $500 \mathrm{kA}$.

A helical deformation of the axis in excess of $10 \%$ of the minor radius develops in the range $0.95 \leqslant q_{\min } \leqslant 1.01$. Only the axisymmetric branch solution exists for $q_{\min }>1.025$. However, a weak helical distortion of the magnetic axis persists for $q_{\min }<0.95$.

\section{Conclusions and discussion}

Three-dimensional bifurcated equilibrium states with helical core structures have been computed in a fixed boundary JET configuration that can model the snakes observed in the experiment and a free boundary TCV configuration has been identified that is susceptible to develop internal helical equilibrium structures that could describe the continuous $n=1$ mode that is observed $[4,5]$. The basic condition for helical deformation is a weak reversed core magnetic shear with a value of $q_{\min }$ in the neighbourhood of unity located around or beyond mid-radius. Furthermore, finite $\langle\beta\rangle$ facilitates the formation and enhances the magnitude of the internal excursion of the magnetic axis. 
Two equilibrium solutions are obtained in the range $0.94<q_{\min }<1.03$ for the JET configuration investigated. The axisymmetric branch is shown to be unstable to a $m=1, n=1$ internal kink mode with a linear growth rate that is consistent with the size of the excursion of the magnetic axis in the helical branch, particularly when $q_{\min }>0.96$ (the internal kink remains unstable for $q_{\min }<0.94$ ). The energy differential between the two branches is very small, only fractions of a per cent. Nevertheless, when $q_{\min }<1$, the helical branch has slightly lower energy than its axisymmetric twin. The converse holds when $q_{\min }>1$. We propose that the snake structures observed in JET can be represented by he bifurcated equilibrium we have developed. In this scenario, a peaked pressure is formed on axis by the accumulation of impurities or pellet material which cools the centre of the plasma causing the current channel to become displaced radially outwards. This generates the reversed shear conditions under which the bifurcation takes place. As the range of $q_{\min }$ can exceed unity, our model would be robust to sawtooth events which are observed in the experiment. Furthermore, we have demonstrated that the snake formation at the low end of the $q_{\min }$ range $\left(q_{\min } \sim 0.94\right)$ concentrates well inside the region for which $q_{\min } \leqslant 1$. This could explain the observations on Tore Supra [3].

The free boundary TCV equilibrium states computed with a 3D helical core also display features that look like a snake when we impose a pressure profile that is more peaked than that in the continuous $n=1$ mode that is observed [4,5]. For low $\langle\beta\rangle\left(\beta_{\mathrm{N}}<0.3\right)$, only the axisymmetric branch appears. A bifurcation occurs around $\beta_{\mathrm{N}}=0.3$ and the excursion of the magnetic axis can exceed half the minor radius when $\beta_{\mathrm{N}}$ reaches 0.7 , after which it saturates. At $\langle\beta\rangle=1.6 \%$, the $3 \mathrm{D}$ helical core is large in the range $0.95<q_{\min }<1.01$. A weak helical state persits for $q_{\min }<0.95$. The helical structure is strictly internal and does not significantly alter the plasma boundary which is more sensitive to the ripple induced by the discrete toroidal coils in these free boundary calculations.

The emissivity from peaked profiles can highlight the internal structures that are detected. Snakes could thus be investigated in more detail with a wider range of diagnostics than that of the continuous modes reported on TCV $[4,5]$. We contend that the snakes observed on JET, Tore Supra and other machines represent essentially the same physical phenomenon as the TCV continuous modes, the MAST long-lived modes or the NSTX saturated internal kinks. Therefore, for a successful experimental programme, we could envision (1) off-axis electron cyclotron heating and current drive with and without Ohmic current ramp-up to generate the necessary $q$-profile and $\langle\beta\rangle$ that could lead to a 3D helical core structure, (2) injection of Ar impurities to trigger the snake, (3) repetitive current ramp-up and ramp-down to have $q_{\text {min }}$ appear and disappear in a discharge, specifically to move throughout the predicted range for helical deformations to be triggered or removed, and (4) diagnostics like soft and hard $\mathrm{X}$-ray detectors located at different toroidal positions in an attempt to measure snakes, as well as new real time control procedures to optimize the trajectories to quickly identify the best options [31].

The driving motivation for these studies is the susceptibility of ITER hybrid scenario configurations [32,33] to develop 3D internal helical cores [22]. The confinement of $\alpha$-particles within the helical core domain constitutes an issue of real practical importance. The structures we compute do not allow the magnetic field lines to break, precluding the simulation of the dynamics of islands and stochastic regions. The formation of a magnetic island could be very relevant when $q_{\min }<1$, but not for hybrid scenario conditions where $q_{\text {min }} \geqslant 1$. It is conceivable that an equilibrium state with a $q=1$ island has a lower energy than the ideal configuration we calculate. For this purpose, more general equilibrium and stability codes, which are much more time consuming, would be required to assess whether equilibrium structures linked to saturated tearing modes are likely to be formed [14, 15, 34-37]. 


\section{Acknowledgments}

This research was partially sponsored by the Fonds National Suisse de la Recherche Scientifique and Euratom. We are very grateful to S P Hirshman for his invaluable contributions to the development of the $3 \mathrm{D}$ equilibrium solvers we use which has made this research possible.

Euratom (C) 2011.

\section{References}

[1] Weller A et al 1987 Phys. Rev. Lett. 592303

[2] Gill R D, Edwards A W, Pasini D and Weller A et al 1992 Nucl. Fusion 12723

[3] Pecquet A-L et al 1997 Nucl. Fusion 37451

[4] Reimerdes H et al 2006 Plasma Phys. Control. Fusion 481621

[5] Camenen Y et al 2007 Nucl. Fusion 47586

[6] Chapman I T et al 2010 Nucl. Fusion 50045007

[7] Menard J E et al 2005 Nucl. Fusion 45539

[8] Lazarus E A et al 2006 Plasma Phys. Control. Fusion 48 L65

[9] Puiatti M E et al 2009 Plasma Phys. Control. Fusion 51120431

[10] Avinash, Hastie R J, Taylor J B and Cowley S C 1987 Phys. Rev. Lett. 592647

[11] Bussac M N and Pellat R 1987 Phys. Rev. Lett. 592650

[12] Waelbroeck F L 1989 Phys. Fluids B 1499

[13] Charlton L A, Hastie R J and Hender T C 1989 Phys. Fluids B 1798

[14] Lütjens H and Luciani J F 2008 J. Comput. Phys. 2276944

[15] Halpern F D, Leblond D, Lütjens, H and Luciani J F 2011 Plasma Phys. Control. Fusion 53015011

[16] Garabedian 2006 Proc. Natl Acad. Sci. USA 10319232

[17] Hirshman S P and Whitson J C 1983 Phys. Fluids 263553

[18] Hirshman S P and Betancourt O 1991 J. Comput. Phys. 9699

[19] Terranova D et al 2010 Plasma Phys. Control. Fusion 52124023

[20] Cooper W A et al 2009 Comput. Phys. Commun. 1801524

[21] Cooper W A et al 2010 Phys. Rev. Lett. 105035003

[22] Cooper W A, Graves J P and Sauter O 2011 Plasma Phys. Control. Fusion 53024002

[23] Cooper W A et al 2011 Plasma Phys. Control. Fusion 53074008

[24] Cooper W A, Graves J P and Sauter O 2011 Nucl. Fusion 51072002

[25] Chew G F, Goldberger M L and Low F E 1956 Proc. R. Soc. Lond. A 236112

[26] Wesson J A 1995 Plasma Phys. Control. Fusion 37 A337

[27] Aydemir A Y 1987 Phys. Rev. Lett. 59649

[28] Lütjens H, Bondeson A and Sauter O 1996 Comput. Phys. Commun. 9547

[29] Anderson D V, Cooper W A, Gruber R, Merazzi S and Schwenn U 1990 Int. J. Supercomput. Appl. 434

[30] Wesson J A 1986 Plasma Phys. Control. Fusion 28243

[31] Felici F and Sauter O 2011 Nonlinear model-based optimization of actuator trajectories for tokamak plasma profile control Plasma Phys. Control. Fusion 53 submitted

[32] Gormezano C et al 2004 Plasma Phys. Control. Fusion 46 B435

[33] Gormezano C et al 2007 Nucl. Fusion 47 S285

[34] Hirshman S P, Sanchez R and Cook C 2011 Phys. Plasmas 18062504

[35] Reiman A and Pomphrey N 1991 J. Comput. Phys. 94225

[36] Harafuji K, Hayashi T and Sato T 1989 J. Comput. Phys. 8169

[37] Hudson S R, Hole M J and Dewar R L 2007 Phys. Plasmas 14052505 